\title{
Spin Excitations of a Kondo-Screened Atom Coupled to a Second Magnetic Atom
}

\author{
A. F. Otte, ${ }^{1,2, *}$ M. Ternes, ${ }^{1,3}$ S. Loth, ${ }^{1,4}$ C. P. Lutz, ${ }^{1}$ C. F. Hirjibehedin, ${ }^{1,5}$ and A. J. Heinrich ${ }^{1, \dagger}$ \\ ${ }^{1}$ IBM Research Division, Almaden Research Center, San Jose, California 95120, USA \\ ${ }^{2}$ Kamerlingh Onnes Laboratorium, Universiteit Leiden, 2300 RA Leiden, The Netherlands \\ ${ }^{3}$ Max-Planck-Institut für Festkörperforschung, Heisenbergstrasse 1, 70569 Stuttgart, Germany \\ ${ }^{4}$ Department of Physics, University of California at Berkeley, Berkeley, California 94720, USA \\ ${ }^{5}$ London Centre for Nanotechnology, Department of Physics and Astronomy, Department of Chemistry, University College London, \\ London WC1H OAH, United Kingdom
}

(Received 7 April 2009; published 2 September 2009)

\begin{abstract}
Screening the electron spin of a magnetic atom via spin coupling to conduction electrons results in a strong resonant peak in the density of states at the Fermi energy, the Kondo resonance. We show that magnetic coupling of a Kondo atom to another unscreened magnetic atom can split the Kondo resonance into two peaks. Inelastic spin excitation spectroscopy with scanning tunneling microscopy is used to probe the Kondo effect of a $\mathrm{Co}$ atom, supported on a thin insulating layer on a $\mathrm{Cu}$ substrate, that is weakly coupled to a nearby $\mathrm{Fe}$ atom to form an inhomogeneous dimer. The Kondo peak is split by interaction with the non-Kondo atom, but can be reconstituted with a magnetic field of suitable magnitude and direction. Quantitative modeling shows that this magnetic field results in a spin-level degeneracy in the dimer, which enables the Kondo effect to occur.
\end{abstract}

PACS numbers: 75.75.+a, 68.37.Ef

Kondo screening is a many-body phenomenon arising from the interaction between a localized magnetic moment and the conduction electrons in a metal [1]. The Kondo effect has been observed for individual spins in various systems including single magnetic atoms on a metal surface [2-4] and in quantum dots connected to metal leads $[5,6]$. In both cases the effect is evident in transport experiments as a sharp resonance in the density of states at the Fermi energy, which results from a level degeneracy in the ground-state magnetization of the localized spin. If a magnetic field is applied to the spin this degeneracy can be lifted, leading to a splitting of the peak [5-10].

In quantum dots it was shown that a similar splitting of the Kondo peak is observed when a Kondo-screened spin is coupled to a second spin via an exchange interaction [1113]. In this situation, which has been the subject of several theoretical studies [14,15], spin coupling is responsible for lifting the degeneracy in the ground state. It was shown in these systems that if the coupling is antiferromagnetic, an external magnetic field of proper magnitude and direction can cancel the effects of spin coupling and thus re-form the single Kondo peak at the Fermi energy [13].

In recent years, it has become possible to probe the lowenergy spin excitation spectra of magnetic nanostructures at the atomic scale using inelastic spin excitation spectroscopy with a scanning tunneling microscope (STM) [9]. Parameters in model spin Hamiltonians can be determined quantitatively from these spectra [16-19], and clear selection rules derived $[17,20,21]$. Kondo-screened atomic spins are particularly interesting systems to explore with this technique because, unlike spins in artificial quantum dots, they can have larger spin values and are subject to magneto-crystalline anisotropy. Elastic and inelastic tun- neling spectra of these systems can be used to probe the interplay between anisotropy and Kondo screening in an environment that can be characterized with atomic-scale precision [10].

In this Letter we use an STM to study interactions in a dimer formed from a Kondo-screened atomic spin and a magnetic atom that is not Kondo screened. Conductance spectra show clearly that there is spin coupling between the two atoms, but we find that the Kondo resonance is localized to the Kondo-screened atom. This resonance is split in the absence of a magnetic field but can be reformed through the application of a field of suitable magnitude and direction. We show that these results can be understood quantitatively by including an isotropic Heisenberg exchange coupling between the two spins. This provides a deep understanding of the interplay between Kondo screening, exchange coupling, and magneto-crystalline anisotropy in this most basic realization of a two magnetic impurity system. Furthermore, this system has the potential to be scaled up to build a variety of interesting lattice structures.

Individual $\mathrm{Co}$ and $\mathrm{Fe}$ atoms were placed onto a $\mathrm{Cu}_{2} \mathrm{~N} / \mathrm{Cu}(100)$ surface [22] by thermal evaporation from pure metal sources. Fe and Co atoms were repositioned, and dimers consisting of one atom of each type were assembled using vertical atom manipulation [16]. The structures were built on clean $\mathrm{Cu}_{2} \mathrm{~N}$ islands and care was taken that no defect, island edge or other adatom was present within 4 lattice constants (i.e., $1.44 \mathrm{~nm}$ ) of any of the atoms probed.

Tunneling spectra of the atoms at $0.5 \mathrm{~K}$ were obtained by measuring the differential conductance $d I / d V$, where $I$ is the current, as a function of the sample voltage $V$ using 
lock-in detection with a $50 \mu \mathrm{V}$ AC modulation at $745 \mathrm{~Hz}$. Inelastic spin excitations can be recognized as distinct upward steps in the spectra [9] where the value of $V$ at the step gives the energy of the excitation. The spectrum for an isolated $\mathrm{Fe}$ atom (i.e., far from any other adatom or island edge) shows steps at three energies (Fig. 1) as observed previously [17]. Spectra of an isolated Co atom also show conductance steps, and additionally show a prominent conductance peak at the Fermi energy $(V=0)$ due to a Kondo resonance with a Kondo temperature of $2.6 \mathrm{~K}[10]$.

The $\mathrm{Co}$ and $\mathrm{Fe}$ atoms were positioned on $\mathrm{Cu}$ binding sites separated by $0.72 \mathrm{~nm}$ (right inset of Fig. 1). This structure was chosen to give a coupling strength comparable to accessible Zeeman energies. Figure 1 shows spectra measured with the tip positioned over the Fe and over the Co atom of the dimer structure. The shapes of the spectra remain recognizably similar to spectra for the isolated atoms, but the Kondo peak in the Co curve has split, even in the absence of a magnetic field. Additionally, changes in the positions and intensities (step heights) of some of the spin excitation steps are observed.

In order to characterize the influence of spin coupling in the dimer on the Kondo screening, we applied a magnetic field along the $x$ and $y$ axes, which are the two highsymmetry in-plane directions (Fig. 2). A magnetic field applied in the $x$ direction reduces the splitting of the Kondo peaks, and at a field of approximately $2 \mathrm{~T}$ the peaks merge into a single Kondo peak that is similar to the Kondo peak for an isolated Co atom. The $x$ direction is the easy

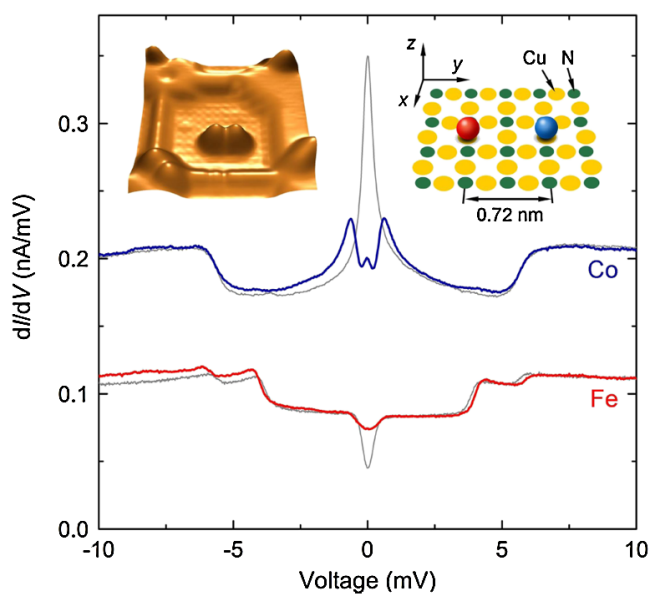

FIG. 1 (color online). Left inset: Topographic STM image $\left(5 \times 5 \mathrm{~nm}^{2}\right)$ of a dimer consisting of an $\mathrm{Fe}$ atom and a Co atom on an island of $\mathrm{Cu}_{2} \mathrm{~N}$ on $\mathrm{Cu}(100)$. The adsorption sites are shown in the right inset. Main panel: Conductance spectra $d I / d V$ taken on the Fe (lower) and Co (upper) atoms in the structure. Light gray curves show respective spectra taken on isolated atoms on $\mathrm{Cu}_{2} \mathrm{~N}$. Both upper curves are offset by $0.1 \mathrm{nA} / \mathrm{mV}$ for clarity. Before taking each spectrum the tip height was adjusted to create a $10 \mathrm{M} \Omega$ tunnel junction (10 mV, $1 \mathrm{nA})$. magnetization direction for $\mathrm{Fe}$ [17] and also lies in the easy magnetization plane for Co [10]. At higher magnetic fields this re-formed Kondo peak splits again into two peaks. In contrast, the splitting increases continuously when a magnetic field is applied along the $y$ direction; in this case there is no field at which a single Kondo peak is formed.

These results suggest that coupling with the Fe spin influences the Co atom as an effective magnetic field along the $x$ direction, which can be cancelled by an external magnetic field. It was shown in [17] that the application of a field of this magnitude along the easy axis of $\mathrm{Fe}$ overcomes the effects of the transverse anisotropy and fully magnetizes the $\mathrm{Fe}$ atom along the direction of the field. This magnetized Fe atom appears to influence the Co atom in a similar fashion as an external magnetic field of approximately $2 \mathrm{~T}$. A comparison between spectra taken on the $\mathrm{Co}$ atom in the dimer and on an isolated Co atom for similar effective fields is presented in Fig. S1 [23].

In order to model the spin excitation energies quantitatively and gain insight into the Kondo screening of the Co atom, we make use of a quantum spin Hamiltonian. A previous study of spin excitations for single Kondoscreened magnetic atoms showed that such a spin Hamiltonian-even though it does not explicitly describe the many-electron effects of the Kondo interactionsquantitatively describes the energies of both the inelastic excitations and of the field-split Kondo peaks [10]. Here we extend this technique to model the coupled-atom system by using the following spin Hamiltonian:
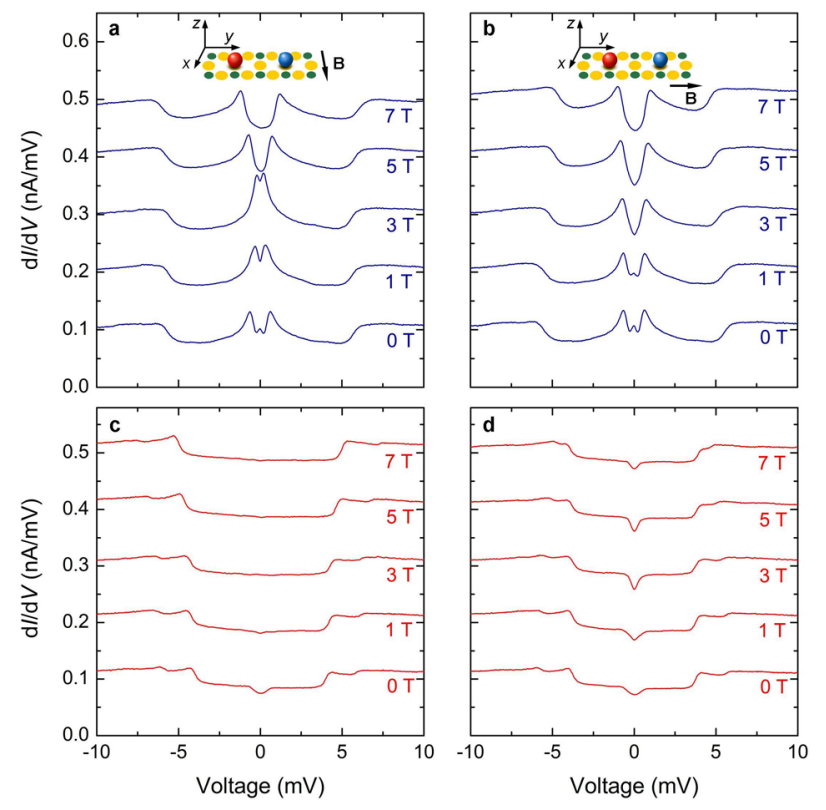

FIG. 2 (color online). Conductance spectra $d I / d V$ measured with the tip positioned over each of the atoms in a dimer, when a magnetic field is applied perpendicular to [(a) for $\mathrm{Co}$, (c) for $\mathrm{Fe}]$ and along the dimer [(b) for $\mathrm{Co}$, (d) for Fe]. Spectra are offset by intervals of $0.1 \mathrm{nA} / \mathrm{mV}$ for clarity. 


$$
\begin{aligned}
\hat{\mathcal{H}}= & J \hat{\mathbf{S}}^{(\mathrm{Fe})} \cdot \hat{\mathbf{S}}^{(\mathrm{Co})}-\mu_{B} \mathbf{B} \cdot\left(g_{\mathrm{Fe}} \hat{\mathbf{S}}^{(\mathrm{Fe})}+g_{\mathrm{Co}} \hat{\mathbf{S}}^{(\mathrm{Co})}\right) \\
& +D_{\mathrm{Fe}} \hat{S}_{x}^{2(\mathrm{Fe})}+E_{\mathrm{Fe}}\left(\hat{S}_{y}^{2(\mathrm{Fe})}-\hat{S}_{z}^{2(\mathrm{Fe})}\right)+D_{\mathrm{Co}} \hat{S}_{y}^{2(\mathrm{Co})}
\end{aligned}
$$

The first term represents an isotropic Heisenberg coupling between the spins $\hat{\mathbf{S}}^{(\mathrm{Fe})}$ on the Fe atom and $\hat{\mathbf{S}}^{(\mathrm{Co})}$ on the Co atom, quantified by the Heisenberg exchange coupling strength $J$. According to this definition, positive values of $J$ signify antiferromagnetic coupling. The second term gives the Zeeman energies resulting from the external magnetic field $\mathbf{B}$, where $\mu_{B}$ denotes the Bohr magneton and $g_{\mathrm{Fe}}$ and $g_{\mathrm{Co}}$ the $g$ factors of the $\mathrm{Fe}$ and Co spins, respectively.

The remaining terms in Eq. (1) represent the magnetocrystalline anisotropies experienced by each of the spins, quantified by the uniaxial anisotropy parameters $D_{\mathrm{Fe}}$ and $D_{\mathrm{Co}}$ and the transverse anisotropy parameter $E_{\mathrm{Fe}}$. All parameters in this spin Hamiltonian except $J$ have been measured previously on the corresponding isolated atoms. The choice of spin magnitudes, $S_{\mathrm{Fe}}=2$ and $S_{\mathrm{Co}}=3 / 2$, the assignment of the axes in the anisotropy terms, and the absence of transverse anisotropy for Co are based on previous studies of the isolated atoms on the same surface $[10,17]$.

Diagonalization of the spin Hamiltonian gives a system of 20 eigenstates with corresponding eigenenergies [24]. To determine which of the excited states are accessible through inelastic excitations by the tunneling electrons we use an excitation intensity model as employed in [17]. Here we extend this model to include the effect of the tip position by using only the spin operators for the atom probed by the tip:

$$
\begin{aligned}
I_{0 \rightarrow n}^{(\mathrm{Co})}= & \left|\left\langle\psi_{n}\left|\hat{S}_{x}^{(\mathrm{Co})}\right| \psi_{0}\right\rangle\right|^{2}+\left|\left\langle\psi_{n}\left|\hat{S}_{y}^{(\mathrm{Co})}\right| \psi_{0}\right\rangle\right|^{2} \\
& +\left|\left\langle\psi_{n}\left|\hat{S}_{z}^{(\mathrm{Co})}\right| \psi_{0}\right\rangle\right|^{2} .
\end{aligned}
$$

Here $I_{0 \rightarrow n}^{(\mathrm{Co})}$ is the transition intensity from the ground state $\left|\psi_{0}\right\rangle$ to the $n$th eigenstate $\left|\psi_{n}\right\rangle$ when the tip is over the Co atom. Note that the wave functions $\left|\psi_{i}\right\rangle$ encode the spin states of both atoms. A similar transition intensity formula using the $\mathrm{Fe}$ spin operators applies for the tip positioned over the Fe atom.

In order to determine the parameters of the spin Hamiltonian we calculated the eigenenergies and the corresponding transition intensities of the dimer for various magnetic fields. Best agreement with the measured step and peak energies, taken from Fig. 2, is achieved by using an antiferromagnetic exchange coupling of strength $J=$ $0.13 \pm 0.02 \mathrm{meV}$ [25].

Measured step and peak energies for both the $\mathrm{Co}$ and the $\mathrm{Fe}$ atom are shown together with the calculated energies in Fig. 3 for fields along $x$ and Fig. S2 for fields along $y$ [23]. There is excellent agreement between the experimental data and the eigenenergies of the spin Hamiltonian and their corresponding intensities: measured data-points ap-

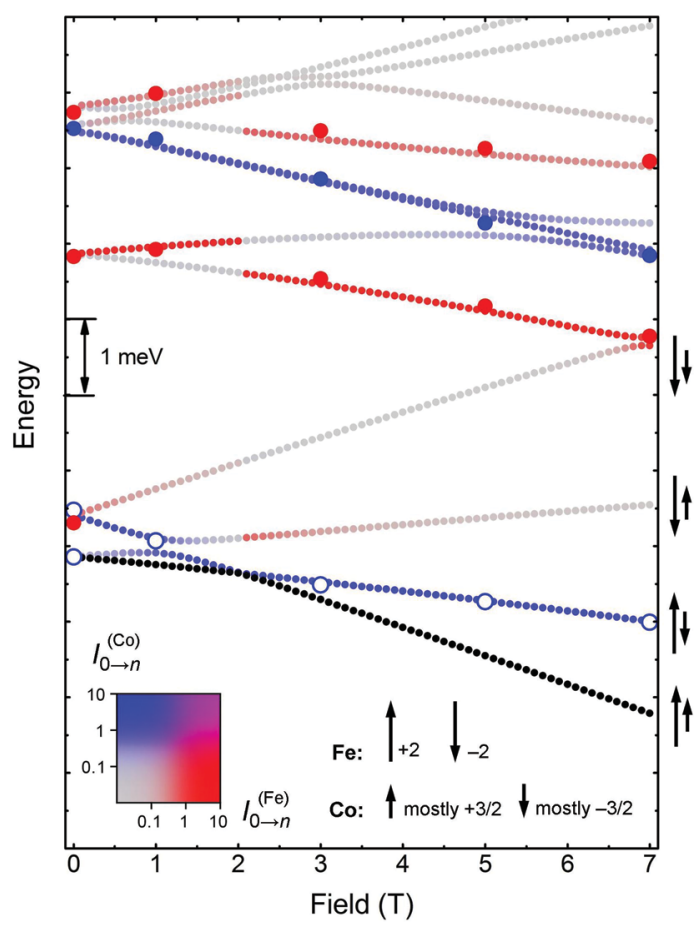

FIG. 3 (color). Small dots: lowest 12 eigenvalues of Eq. (1) with $J=0.13 \mathrm{meV}, \quad g_{\mathrm{Fe}}=2.11, \quad g_{\mathrm{Co}}=2.16, \quad D_{\mathrm{Fe}}=$ $-1.53 \mathrm{meV}, E_{\mathrm{Fe}}=0.31 \mathrm{meV}$ and $D_{\mathrm{Co}}=2.70 \mathrm{meV}$ for $B=0$ to $7 \mathrm{~T}$ in increments of $0.1 \mathrm{~T}$ along $x$. Color indicates the values of $I_{0 \rightarrow n}^{(\mathrm{Co})}$ (blue) and $I_{0 \rightarrow n}^{(\mathrm{Fe})}$ (red) as indicated by the color key on the left. The ground state is colored black. Large dots: experimental data points indicating positions of peaks (open circles) and steps (filled circles) in Figs. 2(a) (blue, Co) and 2(c) (red, Fe), all plotted relative to the calculated ground state. The zero of the energy axis is arbitrary, as only energy differences between states are observable. Arrows indicate the magnetization along the $x$ direction of the Co spin (short arrows) and the Fe spin (long arrows) for the four lowest-energy states. This labeling also applies at low fields, as if the avoided crossing near $1 \mathrm{~T}$ were a true crossing. At the ground-state level crossing at $2.1 \mathrm{~T}$, only the Co spin changes direction.

pear only where the energy level has a large calculated intensity for the spin excitation to that level.

The lowest four eigenstates of the dimer can be understood qualitatively: they consist of two doublets, one doublet where the $x$ component of the Co spin is mostly antiparallel to the Fe spin, and one where it is mostly parallel, as depicted schematically by arrows in Fig. 3. These doublets are offset from each other at zero field by an amount proportional to the coupling strength $J$.

The calculation of the eigenenergies of the spin Hamiltonian explains the reemergence of a single Kondo peak on the Co atom near $B=2 \mathrm{~T}$. According to the model calculation, at $B=2.1 \mathrm{~T}$ the ground and first excited states become degenerate, and for higher magnetic fields the order of the states changes; hence a true level crossing is observed at this field. Therefore, at this level crossing two essential conditions for the occurrence of a 
Kondo system are fulfilled: first, there is a degeneracy between two states, and second, these degenerate states have allowed electronic transitions between them due to flipping the spin of an electron, as described by the equation for $I_{0 \rightarrow n}^{(\mathrm{CO})}$. At magnetic fields near this level crossing, the Fe spin remains essentially fixed at maximum magnetization $\left(m_{\mathrm{Fe}}=+2\right)$ along $x$ in both these states. The ground state changes from the Co spin being mostly antiparallel to the Fe spin for magnetic fields just below the crossing, to mostly parallel.

It is interesting that while the preferred direction of the Fe spin in the ground state does not change at the level crossing, there is a change in what excitations are observable at the $\mathrm{Fe}$ atom. Figure 3 shows that for the excitations at approximately $4 \mathrm{meV}$ and approximately $6 \mathrm{meV}$ above the ground state (which are excitations to $\left|m_{\mathrm{Fe}}\right|=1$ states), the measured step energy suddenly decreases, which agrees well with the calculation.

The observed isotropic Heisenberg exchange coupling $J=0.13 \mathrm{meV}$ is very likely mediated by the $\mathrm{Cu}_{2} \mathrm{~N}$ surface. This is apparent since dimers built with the same spacing of $0.72 \mathrm{~nm}$ but placed so two $\mathrm{N}$ atoms lie between them, show an approximately 10 times stronger coupling than the ones discussed here. This is in general agreement with the observation from density functional studies that the magnetic atoms couple strongly through the $\mathrm{N}$ atoms because they are incorporated in a surface molecular network rather than simply sitting on top of the surface [17]. We note that although the data presented here is consistent with the assumption of an isotropic interaction, we cannot exclude the possibility of an anisotropic interaction.

The success of the spin Hamiltonian used here in modeling the Kondo peak splitting further strengthens the previous observation that the Kondo peak splits in energy in accordance with the underlying non-Kondo physics $[5,6,10]$. As discussed above, the effect of the spin coupling between the atoms is cancelled by the proper magnetic field, and the electrons in the conduction searesponsible for the Kondo screening of the Co atom-are free to cause zero-energy spin-flip transitions of the Co spin. At this field, the measured conductance spectrum is nearly identical to the spectrum of an isolated Co atom at zero field (see Fig. S1 [23]). This is surprising as one might expect that the many-body physics of the Kondo system could behave in a dramatically different way.

The zero-field spectrum of the Co atom in the dimer of Fig. 1 shows an additional narrow peak at $V=0$. There is a twofold degeneracy in the dimer's ground state (Fig. 3), so we speculate that this small conductance peak may be a Kondo resonance of the combined spin of the dimer. Exciting extensions of the presented work are to Kondo dimers and to Kondo lattices, where the proper tuning of the relative strength of the Kondo interaction and the dimer exchange coupling is expected to give rise to a quantum phase transition [26].
We thank B.A. Jones and J.M. van Ruitenbeek for stimulating discussions and B.J. Melior for his expert technical assistance. A. F. O. acknowledges support from the Leiden University Fund; S. L. from the Alexander von Humboldt Foundation and NSF GOALI grant; C.F.H. from EPSRC Grant No. EP/D063604/1; and M.T., C.P. L., and A. J.H. from the Office of Naval Research.

*Present address: Center for Nanoscale Science and Technology, NIST, Gaithersburg, MD 20899, USA †heinrich@almaden.ibm.com

[1] A. C. Hewson, The Kondo Problem to Heavy Fermions (Cambridge University Press, Cambridge, UK, 1997).

[2] V. Madhavan, W. Chen, T. Jamneala, M. F. Crommie, and N. S. Wingreen, Science 280, 567 (1998).

[3] J. T. Li, W. D. Schneider, R. Berndt, and B. Delley, Phys. Rev. Lett. 80, 2893 (1998).

[4] M. Ternes, A. J. Heinrich, and W. D. Schneider, J. Phys. Condens. Matter 21, 053001 (2009).

[5] D. Goldhaber-Gordon et al., Nature (London) 391, 156 (1998).

[6] S.M. Cronenwett, T. H. Oosterkamp, and L.P. Kouwenhoven, Science 281, 540 (1998).

[7] J. Park et al., Nature (London) 417, 722 (2002).

[8] W. Liang, M. P. Shores, M. Bockrath, J. R. Long, and H. Park, Nature (London) 417, 725 (2002).

[9] A. J. Heinrich, J. A. Gupta, C. P. Lutz, and D. M. Eigler, Science 306, 466 (2004).

[10] A. F. Otte et al., Nature Phys. 4, 847 (2008).

[11] N. J. Craig et al., Science 304, 565 (2004).

[12] J. C. Chen, A. M. Chang, and M. R. Melloch, Phys. Rev. Lett. 92, 176801 (2004).

[13] H. B. Heersche et al., Phys. Rev. Lett. 96, 017205 (2006).

[14] P. Simon, R. Lopez, and Y. Oreg, Phys. Rev. Lett. 94, 086602 (2005).

[15] M. G. Vavilov and L. I. Glazman, Phys. Rev. Lett. 94, 086805 (2005).

[16] C. F. Hirjibehedin, C. P. Lutz, and A. J. Heinrich, Science 312, 1021 (2006).

[17] C. F. Hirjibehedin et al., Science 317, 1199 (2007).

[18] X. Chen et al., Phys. Rev. Lett. 101, 197208 (2008).

[19] N. Tsukahara et al., Phys. Rev. Lett. 102, 167203 (2009).

[20] M. Persson, Phys. Rev. Lett. 103, 050801 (2009).

[21] J. Fernandez-Rossier, Phys. Rev. Lett. 102, 256802 (2009).

[22] T. Choi, C. D. Ruggiero, and J. A. Gupta, Phys. Rev. B 78, 035430 (2008).

[23] See EPAPS Document No. E-PRLTAO-103-068937. For more information on EPAPS, see http://www.aip.org/ pubservs/epaps.html.

[24] R. I. Nepomechie, Int. J. Mod. Phys. B 13, 2973 (1999).

[25] Values for $g_{\mathrm{Fe}}, g_{\mathrm{Co}}, D_{\mathrm{Fe}}, E_{\mathrm{Fe}}$, and $D_{\mathrm{Co}}$ were optimized to fit the measured spectra, and differ by less than $5 \%$ from each of the values found previously for the corresponding isolated atoms [10,17].

[26] I. Affleck, A. W. W. Ludwig, and B. A. Jones, Phys. Rev. B 52, 9528 (1995). 